\section{Alimentação fora de casa e biomarcadores de doenças crônicas em adolescentes brasileiros}

\author{
Eating away from home and biomarkers for \\ chronic noncommunicable diseases in \\ Brazilian adolescents
}

\section{Alimentación fuera de casa y biomarcadores de enfermedades crónicas en adolescentes brasileños}

\author{
Suelyne Rodrigues de Morais 1 \\ Ilana Nogueira Bezerra 1 \\ Amanda de Moura Souza 2 \\ Clarice Maria Araújo Chagas Vergara 1 \\ Rosely Sichieri 3
}

doi: 10.1590/0102-311X00219619

\title{
Resumo
}

Objetivou-se avaliar a relação entre o consumo de alimentos fora de casa e alterações em biomarcadores de doenças crônicas em adolescentes brasileiros. Trata-se de um estudo transversal em que foram utilizados os dados do Estudo de Riscos Cardiovasculares em Adolescentes (ERICA), conduzido com 36.956 adolescentes, em 2013/2014. A relação entre consumir alimentos fora de casa e cada desfecho de interesse (hipertrigliceridemia, hipercolesterolemia, hiperglicemia, elevada hemoglobina glicada e hiperinsulinemia) foi testada por modelos de regressão logística ajustados por idade, rede de ensino da escola, atividade física e tempo de uso de telas. Dados de consumo alimentar foram obtidos pela aplicação do recordatório referente às $24 \mathrm{~h}$ anteriores (R24h), analisando o consumo de energia, açúcar de adição, sódio, potássio, fibras, frutas, verduras, arroz, feijão, sanduíches, bolos, sobremesas, chocolates e refrigerantes. Foi encontrado que 53,2\% dos adolescentes consumiam alimentos fora de casa. A alimentação fora de casa apresentou relação inversa com hiperinsulinemia $(O R=0,65$; IC95\%: 0,46-0,92) e hiperglicemia $(O R=0,46$; IC95\%: 0,30-0,71), entre meninos, e hipertensão $(O R=0,71$; IC95\%: 0,55-0,92) e hiperglicemia $(O R=0,57$; IC95\%: 0,34-0,96), entre meninas. Entretanto, o consumo de calorias, açúcar de adição, sanduíches, sobremesas e refrigerantes foi maior nos adolescentes que consumiam alimentos fora de casa. O papel protetor da alimentação fora de casa em indicadores bioquímicos nos adolescentes pode ser em função de um maior consumo da alimentação escolar, mais frequente entre os adolescentes que consumiam alimentos fora de casa, destacando a importância do estímulo ao consumo da alimentação escolar.

Comportamento Alimentar; Estilo de Vida; Hipertensão; Diabetes Mellitus; Doenças Não Transmissíveis

\author{
Correspondência \\ S. R. Morais \\ Universidade Estadual do Ceará. \\ Av. Doutor Silas Munguba 1700, Fortaleza, CE \\ 60741-000, Brasil. \\ suelyne_rodrigues@hotmail.com \\ 1 Universidade Estadual do Ceará, Fortaleza, Brasil. \\ 2 Universidade Federal do Rio de Janeiro, Rio de Janeiro, Brasil. \\ 3 Instituto de Medicina Social, Universidade do Estado do Rio de \\ Janeiro, Rio de Janeiro, Brasil.
}




\section{Introdução}

A distinção dos efeitos entre comportamentos específicos para a ocorrência de excesso de peso e doenças crônicas não transmissíveis (DCNT) é um desafio, particularmente na adolescência, na qual o ganho de peso faz parte do desenvolvimento natural desse período. A realização de refeições fora do lar destaca-se como um comportamento que favorece o consumo excessivo de alimentos ultraprocessados e energia, e, consequentemente, o ganho de peso acima do recomendado e o desenvolvimento de DCNT 1,2,3.

Dadas as características nutricionais dos alimentos consumidos fora de casa, é razoável supor que os biomarcadores de doenças crônicas possam favorecer a compreensão de como o consumo de alimentos fora de casa medeia desfechos desfavoráveis à saúde entre os adolescentes. No entanto, poucos estudos têm discutido essa questão, e nenhum foi realizado em uma amostra representativa da população brasileira, que apresenta características bem diferentes de adolescentes de países de alta renda, onde a maioria desses estudos têm sido realizados 4,5,6,7.

A importância de se avaliar o consumo de alimentos fora de casa em adolescentes é que esse tende a aumentar com a idade em crianças, sendo o pico na adolescência tardia (15 a 18 anos), podendo perdurar na vida adulta 8 . No Brasil, $48 \%$ dos adolescentes reportaram o consumo de, pelo menos, um item fora de casa em um dia de registro alimentar em 2008-2009 9.

As poucas publicações sobre o consumo de alimentos fora do lar em adolescentes brasileiros limitam-se a descrever as frequências de consumo nessa população e a contribuição da alimentação fora de casa para o consumo de itens específicos 9. Não há estudos que tenham investigado a associação entre consumo de alimentos fora de casa e marcadores bioquímicos de doenças crônicas na população brasileira, apesar de já se saber que altos níveis de biomarcadores para essas doenças na adolescência estão associados a uma maior chance de desenvolvimentos das DCNT na vida adulta 10,11.

O presente estudo apresenta a avaliação da relação entre o consumo de alimentos fora de casa e alterações em biomarcadores de doenças crônicas em adolescentes brasileiros.

\section{Métodos}

Trata-se de um estudo transversal em que se utilizou dos dados provenientes do Estudo de Riscos Cardiovasculares em Adolescentes (ERICA), conduzido em 2013-2014. O ERICA é um estudo multicêntrico brasileiro de amplitude nacional de base escolar, que teve como objetivo conhecer a proporção de adolescentes com diabetes e obesidade e traçar o perfil dos fatores de risco para doenças cardiovasculares em adolescentes brasileiros de 12 a 17 anos, que estudavam nas três últimas séries do ensino fundamental ou nas três séries do ensino médio em escolas públicas e privadas de municípios com mais de 100 mil habitantes, de todas as cinco macrorregiões do Brasil 12.

Resumidamente, o ERICA adotou um plano amostral em três estágios. No primeiro estágio, a população do estudo foi estratificada em 32 estratos, um para cada uma das 27 capitais brasileiras e mais cinco estratos, advindos de conjuntos de municípios com população acima de 100 mil habitantes. No total, foram avaliadas 1.247 escolas distribuídas em 122 municípios. Para o segundo estágio amostral, foram selecionadas três turmas de cada escola, considerando-se os turnos manhã e tarde e anos elegíveis (do sétimo ano do Ensino Fundamental ao terceiro ano do Ensino Médio). O terceiro estágio compreendeu os adolescentes das turmas selecionadas. Para o ERICA, foram incluídos todos os adolescentes sem deficiência física provisória ou permanente e as adolescentes que não estavam grávidas. Dos 102.237 adolescentes elegíveis, 72.508 estudavam de manhã e foram selecionados devido à necessidade do jejum para a coleta dos exames de sangue. Os participantes foram agrupados em subconjuntos de acordo com a completude das informações, sendo o peso amostral calculado para cada um dos subconjuntos definidos. Para este estudo, foram avaliados 36.956 adolescentes, que responderam o recordatório alimentar referente às 24 horas anteriores (R24h), o questionário do adolescente, realizaram exames de sangue e antropometria e aferiram a pressão arterial 13,14.

Para a coleta de dados do ERICA, os avaliadores foram previamente treinados utilizando técnicas padronizadas. Os adolescentes responderam um questionário autopreenchido com 11 blocos de perguntas envolvendo dados socioeconômicos e de estilo de vida, usando-se um coletor eletrônico de 
dados (o personal digital assistant - PDA) e um recordatório alimentar referente às 24 horas anteriores. Tiveram suas medidas antropométricas e pressão arterial aferidas e realizaram exames sanguíneos em jejum: triglicerídeos, LDLc (colesterol associado à lipoproteína de baixa densidade), HDLc (colesterol associado à lipoproteína de alta densidade), colesterol total, glicose, hemoglobina glicada e insulina 15.

Para a verificação das medidas antropométricas e da pressão arterial, utilizou-se a metodologia citada por Bloch et al. 15. O peso foi obtido em balança eletrônica da marca Líder Balanças (Araçatuba, Brasil) com capacidade de $200 \mathrm{~kg}$ e variação de $50 \mathrm{~g}$. A estatura foi medida utilizando-se estadiômetro portátil e desmontável da marca Alturexata (Belo Horizonte, Brasil), com resolução de $1 \mathrm{~mm}$ e campo de uso de até $213 \mathrm{~cm}$. Calculou-se o índice de massa corporal (IMC), e o estado nutricional dos adolescentes foi avaliado utilizando-se as curvas propostas pela Organização Mundial da Saúde (OMS), de 2007, usando, como índice, o IMC-para-idade, segundo sexo. Os adolescentes foram classificados em tendo excesso de peso quando o IMC-para-idade apresentou-se acima de +1 z-escore 16 .

Aferiu-se a pressão arterial com o aparelho da marca Omron (São Paulo, Brasil), modelo 705-IT, validado para uso em adolescentes. Os adolescentes foram classificados como hipertensos quando apresentaram a pressão arterial sistólica ou a diastólica correspondente ao percentil 95 ou superior para sua estatura, sexo e idade 15.

Todas as análises laboratoriais foram realizadas em um único laboratório, seguindo normas de qualidade vigentes e exigidas para sua qualificação. Os adolescentes foram orientados a ficar em jejum por 12 horas antes da coleta. Foram seguidos os pontos de corte da V Diretriz Brasileira de Dislipidemias e Prevenção da Aterosclerose (2013) para classificar os adolescentes quanto à alteração dos exames bioquímicos 17 .

Os pontos de corte considerados elevados ou não desejáveis para avaliação dos exames bioquímicos foram: triglicerídeos $\geq 130 \mathrm{mg} / \mathrm{dL}$, colesterol total $\geq 170 \mathrm{mg} / \mathrm{dL}, \mathrm{LDL}-\mathrm{c} \geq 130 \mathrm{mg} / \mathrm{dL}$, HDL-c < $45 \mathrm{mg} / \mathrm{dL}$, insulina $\geq 20 \mathrm{mU} / \mathrm{L}$, glicemia $\geq 100 \mathrm{mg} / \mathrm{dL}$ e a hemoglobina glicada $\geq 5,7 \% 17,18,19$. A descrição completa do método pode ser vista em Bloch et al. 15 .

Os dados de consumo alimentar foram obtidos pela aplicação do R24h do dia anterior. Dois recordatórios foram aplicados em dias não consecutivos, no entanto, o segundo foi aplicado somente em 7\% da amostra. Para este estudo, utilizamos o primeiro dia do R24h. Os adolescentes foram entrevistados por pesquisadores de campo treinados, que utilizaram software específico para a entrada de dados de consumo alimentar ${ }^{20}$, com registro direto das informações em computadores, utilizando o multiple-pass method com objetivo de reduzir o sub-relato do consumo de alimentos 21 . Os entrevistadores registraram todos os alimentos e as bebidas consumidos pelos participantes no dia anterior à entrevista, o local de consumo dos itens (em casa, na escola e em outros lugares) e o horário em que foram consumidos 15 .

Os indivíduos foram classificados como consumidores de alimentos fora de casa se tiverem apresentado o consumo de, pelo menos, um item fora de casa, independentemente de o consumo ter sido feito na escola ou não, e a variável de exposição foi o consumo de alimentos fora de casa (sim/não).

Os desfechos analisados foram excesso de peso, hipertensão e alterações em marcadores bioquímicos (glicose, insulina, hemoglobina glicada, triglicerídeos, colesterol total, LDL-c e HDL-c), conforme pontos de corte descritos acima.

Outras variáveis incluídas nas análises foram: faixa etária (12 a 14 anos ou 15 a 17 anos), sexo (feminino ou masculino), rede de ensino da escola (pública ou privada), comportamento sedentário (foi considerado sedentário o indivíduo em que o tempo de uso de computador, televisão ou videogame em um dia de semana comum foi de duas horas ou mais por dia e não sedentário aquele com menos de duas horas por dia) e prática de atividade física em que foram considerados ativos quando a atividade física correspondeu a 300 minutos ou mais por semana e inativos quando a atividade física foi inferior a 300 minutos por semana, de acordo com os pontos de corte estabelecidos por Hallal et al. 22 .

As análises foram realizadas com o software SAS Studio (https://www.sas.com/pt_br/software/ studio.html), utilizando os procedimentos survey para incorporação da complexidade da amostra. Calculou-se a frequência de indivíduos que consomem alimentos fora do domicílio segundo sexo, faixas de idade, rede de ensino da escola, tempo de uso de telas e nível de atividade física. Estimou-se a prevalência de hipertensão e inadequação dos marcadores bioquímicos entre consumidores e não consumidores de alimentos fora de casa. Todas as frequências foram apresentadas separadamente para meninos e meninas e com os respectivos intervalos de 95\% de confiança (IC95\%). 
A relação entre consumir alimentos fora de casa (variável independente) e cada desfecho de interesse (elevada pressão arterial e alteração não desejável dos marcadores bioquímicos (variáveis dependentes) foi testada por análise multivariada, utilizando regressão logística ajustada por meio de três modelos: modelo 1 (idade), modelo 2 (idade e rede de ensino da escola) e modelo 3 (idade, rede de ensino da escola, atividade física e tempo de uso de telas). Análises adicionais excluíram da alimentação fora de casa os consumos realizados na escola. Ainda, a ingestão total de energia (Kcal/dia), açúcar de adição (percentual com relação ao valor calórico total da dieta), sódio (mg/1.000Kcal), potássio (mg/1.000Kcal), relação sódio/potássio, fibras (g/dia) e o consumo de itens específicos marcadores de alimentação saudável (frutas, verduras, arroz e feijão) e não saudável (sanduíches, bolos, sobremesas, chocolates e refrigerantes) também foram analisados. A classificação dos alimentos em marcadores de uma alimentação saudável ou não saudável baseou-se nas recomendações do Guia Alimentar para a População Brasileira de 2014, considerando, como marcadores de uma alimentação saudável, os alimentos in natura e minimamente processados, enquanto alimentos processados e ultraprocessados foram considerados marcadores de uma alimentação não saudável 23. As estimativas foram calculadas entre os que consumiam alimentos fora de casa e os que não consumiam no intuito de avaliar diferenças entre a alimentação realizada dentro e fora de casa.

No intuito de compreender possíveis diferenças entre adolescentes de escolas públicas e privadas, todas as análises foram realizadas e estratificadas por tipo de escola e disponibilizadas em material suplementar.

O ERICA foi conduzido de acordo com a Declaração de Helsinque. O Comitê de Ética em Pesquisa da Universidade Federal do Rio de Janeiro aprovou o estudo em janeiro de 2009, sob número de parecer 01/2009. A aprovação do Comitê de Ética foi obtida em cada um dos 26 estados e para o Distrito Federal. O consentimento informado por escrito foi obtido de cada estudante e de seus pais. Durante toda a coleta e análise de dados, foram tomadas medidas de privacidade e confidencialidade dos alunos.

\section{Resultados}

O consumo de alimentos fora de casa referente às últimas 24 h foi citado por 53,2\% (IC95\%: 50,4-56,1) dos adolescentes. Não houve diferenças nas prevalências de consumo segundo variáveis sociodemográficas, com exceção do tipo de escola, em que os adolescentes de escolas privadas apresentaram maior frequência de consumo de alimentos fora de casa do que os de escolas públicas (Tabela 1).

Diferenças quanto à presença de alterações em marcadores bioquímicos para doenças crônicas foram observadas entre consumidores e não consumidores de alimentos fora de casa. Entre os meninos, os adolescentes que consumiram alimentos fora de casa apresentaram menor prevalência de hiperglicemia se comparados com os meninos que não consumiam alimentos fora de casa. Entretanto, não foram encontradas diferenças significativas nas outras morbidades (hipertensão arterial, hipercolesterolemia, baixo HDL, LDL elevado, hiperinsulinemia, hemoglobina glicada elevada e excesso de peso). Já, entre as meninas, não houve diferença nas prevalências das alterações nesses marcadores, comparando as adolescentes que consumiam alimentos fora de casa e as que não consumiam (Tabelas 2 e 3). Análises estratificadas por tipo de escola mostraram que a maior prevalência de hiperglicemia entre os meninos que não consumiam alimentos fora de casa foi encontrado somente nos meninos das escolas públicas (Material suplementar - Tabelas S1 e S2. http://cadernos.ensp.fiocruz.br/static// arquivo/csp-2196-19-material-suplementar_2353.pdf).

$\mathrm{Na}$ análise de regressão logística, os meninos que consumiam alimentos fora de casa apresentaram menor probabilidade de apresentar hiperinsulinemia e hiperglicemia. Nas meninas, aquelas que consumiam alimentos fora de casa apresentaram menor probabilidade de apresentar hipertensão arterial e hiperglicemia (Tabela 4). A relação inversa entre consumir alimentos fora de casa e hiperinsulinemia e hiperglicemia entre os meninos manteve-se somente entre os adolescentes de escolas públicas. Da mesma forma, o achado de que as meninas que consumiam alimentos fora de casa apresentaram menor probabilidade de apresentar hipertensão arterial e hiperglicemia manteve-se somente entre as meninas das escolas públicas (Material suplementar - Tabela S3. http://cadernos.ensp.fiocruz.br/ static//arquivo/csp-2196-19-material-suplementar_2353.pdf). 
Tabela 1

Prevalências e intervalos de 95\% de confiança (IC95\%) das características sociodemográficas e comportamentais de adolescentes brasileiros, segundo consumo de alimentos fora de casa. Estudo de Riscos Cardiovasculares em Adolescentes (ERICA), Brasil, 2013-2014.

\begin{tabular}{|c|c|c|c|c|}
\hline \multirow[t]{3}{*}{ Características } & \multicolumn{4}{|c|}{ Consumo de alimentos fora de casa } \\
\hline & \multicolumn{2}{|c|}{ Não } & \multicolumn{2}{|c|}{ Sim } \\
\hline & $\mathbf{n}$ & $\%(I C 95 \%)$ & $\mathbf{n}$ & $\%(I C 95 \%)$ \\
\hline \multicolumn{5}{|l|}{ Sexo } \\
\hline Masculino & 6.997 & $48,0(44,2-51,8)$ & 7.789 & $52,0(48,2-55,8)$ \\
\hline Feminino & 9.724 & $45,6(42,6-48,5)$ & 12.446 & $54,4(51,5-57,4)$ \\
\hline \multicolumn{5}{|l|}{ Faixa etária (anos) } \\
\hline $12-14$ & 8.191 & $48,7(43,8-53,6)$ & 8.730 & $51,3(46,4-56,2)$ \\
\hline 15 ou mais & 8.530 & $45,1(42,4-47,7)$ & 11.505 & $54,9(52,3-57,6)$ \\
\hline \multicolumn{5}{|c|}{ Rede de ensino de escola } \\
\hline Pública & 13.244 & $49,4(46,2-52,7)$ & 13.979 & $50,6(47,3-53,8)$ \\
\hline Privada & 3.477 & $37,5(32,7-42,4)$ & 6.256 & $62,5(57,6-67,3)$ \\
\hline \multicolumn{5}{|c|}{ Atividade física (minutos/semana) } \\
\hline$<300$ & 7.909 & $46,9(43,8-50,0)$ & 9.434 & $53,1(50,0-56,2)$ \\
\hline$\geq 300$ & 7.634 & $46,2(43,2-49,1)$ & 9.506 & $53,8(50,9-56,8)$ \\
\hline \multicolumn{5}{|c|}{ Tempo de uso de telas (horas/dia) } \\
\hline$<2$ & 4.027 & $45,5(40,5-50,5)$ & 4.994 & $54,5(49,5-59,5)$ \\
\hline$\geq 2$ & 11.164 & $46,9(43,9-49,8)$ & 13.715 & $53,1(50,2-56,1)$ \\
\hline
\end{tabular}

Tendo em vista que parte do consumo de alimentos fora de casa é decorrente da alimentação escolar, nós avaliamos a oferta e o consumo da alimentação oferecida na escola pelos adolescentes e encontramos que o consumo de alimentação escolar é similar entre os que consomem e não consomem alimentos fora de casa. Por outro lado, a frequência desse consumo é três vezes maior nos adolescentes de escola pública do que nos adolescentes de escolas privadas (61,7\%; IC95\%: 58,8-64,7 vs. 21,4\%; IC95\%: 16,0-26,7).

Em análises adicionais, não classificando os alimentos consumidos na escola como alimentos fora de casa, nós encontramos que 24,5\% (IC95\%: 22,8-26,3) dos adolescentes consumiram alimentos fora de casa. Com essa classificação, não encontramos diferenças sociodemográficas e comportamentais, comparando consumidores e não consumidores de alimentos fora de casa. Quanto às morbidades, resultados similares foram encontrados para hiperglicemia entre os meninos. Os adolescentes que referiram consumir alimentos fora de casa apresentaram menor prevalência de hiperglicemia do que os que não consumiam alimentos fora de casa (3\%; IC95\%: 2,0-4,1 vs. 6,1\%; IC95\%: 4,5-7,6, respectivamente). Em ambos os sexos, a prevalência de hipertensão arterial nos que consumiam alimentos fora de casa foi menor em comparação com quem não referiu consumo de alimentos fora de casa ( $8 \%$; IC95\%: 6,5-9,6 vs. 13,6\%; IC95\%: 12,2-15,0 entre os meninos e 5,4\%; IC95\%: 3,9-7,0 vs. 8,2\%; IC95\%: 7,0-9,4 entre as meninas, respectivamente).

Avaliando a alimentação dentro e fora de casa, a ingestão média calórica e de açúcar de adição foi maior nos adolescentes que consomem alimentos fora de casa, assim como o consumo de sanduíches, sobremesas e refrigerantes (Tabela 5). No entanto, esses achados foram observados somente nas escolas públicas, onde os adolescentes que se alimentavam fora de casa também apresentaram maior consumo de frutas e feijão e menor ingestão de sódio (Material suplementar - Tabela S4. http://cadernos. ensp.fiocruz.br/static//arquivo/csp-2196-19-material-suplementar_2353.pdf). 
Tabela 2

Prevalências e intervalos de 95\% de confiança (IC95\%) de morbidades em adolescentes brasileiros do sexo masculino, segundo consumo de alimentos fora de casa. Estudo de Riscos Cardiovasculares em Adolescentes (ERICA), Brasil, 2013-2014.

\begin{tabular}{|c|c|c|c|c|c|c|}
\hline \multirow[t]{3}{*}{ Morbidades } & \multicolumn{4}{|c|}{ Consumo de alimentos fora de casa } & \multicolumn{2}{|c|}{ Total } \\
\hline & \multicolumn{2}{|r|}{ Não } & \multicolumn{2}{|r|}{ Sim } & \multirow[b]{2}{*}{$\mathbf{n}$} & \multirow[b]{2}{*}{$\%$ (IC95\%) } \\
\hline & $\mathbf{n}$ & $\%$ (IC95\%) & $\mathbf{n}$ & $\%($ IC95\%) & & \\
\hline \multicolumn{7}{|l|}{ Hipertensão arterial } \\
\hline Não hipertenso & 6.022 & $86,6(84,8-88,5)$ & 6.910 & $88,7(86,7-90,7)$ & 12.932 & $87,7(86,6-88,8)$ \\
\hline Hipertenso & 976 & $13,4(11,5-15,2)$ & 878 & $11,3(9,3-13,3)$ & 1.854 & $12,3(11,2-13,4)$ \\
\hline \multicolumn{7}{|l|}{ Colesterol } \\
\hline Adequado & 5.911 & $85,2(83,4-87,0)$ & 6.545 & $83,9(81,5-86,4)$ & 12.456 & $84,5(82,8-86,3)$ \\
\hline Elevado & 1.065 & $14,8(13,0-16,6)$ & 1.225 & $16,1(13,6-18,5)$ & 2.290 & $15,5(13,7-17,2)$ \\
\hline \multicolumn{7}{|l|}{ HDLC } \\
\hline Adequado & 3.034 & $43,5(40,5-46,5)$ & 3.373 & $44,9(40,7-49,1)$ & 6.407 & $44,2(41,7-46,7)$ \\
\hline Não desejável & 3.943 & $56,5(53,4-59,5)$ & 4.396 & $55,1(50,9-59,3)$ & 8.339 & $55,8(53,3-58,3)$ \\
\hline \multicolumn{7}{|l|}{ LDLc } \\
\hline Adequado & 6.748 & $97,3(96,4-98,1)$ & 7.539 & $97,1(96,3-97,9)$ & 14.287 & $97,2(96,5-97,9)$ \\
\hline Elevado & 224 & $2,7(1,9-3,6)$ & 225 & $2,9(2,1-3,7)$ & 449 & $2,8(2,1-3,5)$ \\
\hline \multicolumn{7}{|l|}{ Triglicerídeos } \\
\hline Adequado & 6.467 & $92,7(91,4-94,0)$ & 7.176 & $92,3(90,2-94,3)$ & 13.643 & $92,5(91,2-93,7)$ \\
\hline Elevado & 531 & $7,3(6,0-8,6)$ & 612 & $7,7(5,7-9,8)$ & 1.143 & $7,5(6,3-8,8)$ \\
\hline \multicolumn{7}{|l|}{ Insulina } \\
\hline Adequado & 6.639 & $95,0(93,8-96,2)$ & 7.463 & $96,5(95,6-97,4)$ & 14.112 & $95,8(94,9-96,6)$ \\
\hline Elevado & 319 & $5,0(3,8-6,2)$ & 298 & $3,5(2,6-4,4)$ & 617 & $4,2(3,4-5,1)$ \\
\hline \multicolumn{7}{|l|}{ Glicemia } \\
\hline Adequado & 6.606 & $92,7(90,7-94,8)$ & 7.488 & $96,5(95,5-97,4)$ & 14.094 & $94,7(93,5-95,9)$ \\
\hline Elevado & 352 & $7,3(5,2-9,3)$ & 274 & $3,5(2,6-4,5)$ & 626 & $5,3(4,1-6,5)$ \\
\hline \multicolumn{7}{|l|}{ HBG } \\
\hline Adequado & 5.989 & $86,0(83,5-88,5)$ & 6.673 & $84,7(82,8-86,7)$ & 12.662 & $85,3(83,7-87,0)$ \\
\hline Não desejável & 1.005 & $14,0(11,5-16,5)$ & 1.103 & $15,3(13,3-17,2)$ & 2.108 & $14,7(13,0-16,3)$ \\
\hline \multicolumn{7}{|l|}{ Excesso de peso } \\
\hline Não & 5.093 & $73,4(71,1-75,8)$ & 5.666 & $71,6(67,9-75,3)$ & 10.759 & $72,5(70,2-74,7)$ \\
\hline Sim & 1.905 & $26,6(24,2-28,9)$ & 2.122 & $28,4(24,7-32,1)$ & 4.027 & $27,5(25,3-29,8)$ \\
\hline
\end{tabular}

HBG: hemoglobina glicada; HDLc: colesterol associado à lipoproteína de alta densidade; LDLc: colesterol associado à lipoproteína de baixa densidade. 
Tabela 3

Prevalências e intervalos de 95\% de confiança (IC95\%) de morbidades em adolescentes brasileiros do sexo feminino, segundo consumo de alimentos fora de casa. Estudo de Riscos Cardiovasculares em Adolescentes (ERICA), Brasil, 2013-2014.

\begin{tabular}{|c|c|c|c|c|c|c|}
\hline \multirow[t]{3}{*}{ Morbidades } & \multicolumn{4}{|c|}{ Consumo de alimentos fora de casa } & \multicolumn{2}{|r|}{ Total } \\
\hline & \multicolumn{2}{|r|}{ Não } & \multicolumn{2}{|r|}{ Sim } & \multirow[b]{2}{*}{$\mathrm{n}$} & \multirow[b]{2}{*}{$\%$ (IC95\%) } \\
\hline & n & $\%($ IC95\%) & $\mathbf{n}$ & $\%$ (IC95\%) & & \\
\hline \multicolumn{7}{|l|}{ Hipertensão arterial } \\
\hline Não hipertenso & 8.942 & $91,1(89,6-92,7)$ & 11.719 & $93,6(92,6-94,6)$ & 20.661 & $92,5(91,5-93,5)$ \\
\hline Hipertenso & 782 & $8,9(7,3-10,4)$ & 727 & $6,4(5,4-7,4)$ & 1.509 & $7,5(6,5-8,5)$ \\
\hline \multicolumn{7}{|l|}{ Colesterol } \\
\hline Adequado & 7.373 & $74,1(70,9-77,4)$ & 9.425 & $75,6(73,9-77,4)$ & 16.798 & $75,0(73,3-76,6)$ \\
\hline Elevado & 2.321 & $25,9(22,6-29,1)$ & 2.986 & $24,4(22,6-26,1)$ & 5.487 & $25,0(23,4-26,7)$ \\
\hline \multicolumn{7}{|l|}{ HDLC } \\
\hline Adequado & 5.710 & $61,4(57,6-65,1)$ & 7.589 & $63,4(60,4-66,5)$ & 13.299 & $62,5(59,7-65,3)$ \\
\hline Não desejável & 3.984 & $38,6(34,9-42,4)$ & 4.822 & $36,6(33,5-39,6)$ & 8.806 & $37,5(34,7-40,3)$ \\
\hline \multicolumn{7}{|l|}{ LDLC } \\
\hline Adequado & 9.277 & $95,8(94,8-96,8)$ & 11.871 & $95,7(94,9-96,5)$ & 21.148 & $95,7(95,1-96,4)$ \\
\hline Elevado & 414 & $4,2(3,2-5,2)$ & 539 & $4,3(3,5-5,1)$ & 953 & $4,3(3,6-4,9)$ \\
\hline \multicolumn{7}{|l|}{ Triglicerídeos } \\
\hline Adequado & 8.935 & $91,7(90,2-93,2)$ & 11.460 & $92,5(91,3-93,7)$ & 20.395 & $92,1(91,2-93,1)$ \\
\hline Elevado & 789 & $4,5(6,8-9,8)$ & 986 & $7,5(6,3-8,7)$ & 1.775 & $7,9(6,9-8,8)$ \\
\hline \multicolumn{7}{|l|}{ Insulina } \\
\hline Adequado & 9.137 & $93,5(92,1-95,0)$ & 11.836 & $94,7(93,5-95,9)$ & 20.973 & $94,2(93,0-95,3)$ \\
\hline Elevado & 548 & $6,5(5,0-8,0)$ & 563 & $5,3(4,1-6,5)$ & 1.111 & $5,8(4,7-7,0)$ \\
\hline \multicolumn{7}{|l|}{ Glicemia } \\
\hline Adequado & 9.422 & $96,5(95,2-97,9)$ & 12.171 & $97,9(97,3-98,5)$ & 21.593 & $97,3(96,5-98,0)$ \\
\hline Elevado & 260 & $3,5(2,1-4,8)$ & 223 & $2,1(1,5-2,7)$ & 483 & $2,7(2,0-3,5)$ \\
\hline \multicolumn{7}{|l|}{ HBG } \\
\hline Adequado & 8.737 & $89,0(87,3-90,8)$ & 11.207 & $89,1(87,4-90,7)$ & 19.944 & $89,1(87,7-90,4)$ \\
\hline Não desejável & 971 & $11,0(9,2-12,7)$ & 1.218 & $10,9(9,3-12,6)$ & 2.189 & $10,9(9,6-12,3)$ \\
\hline \multicolumn{7}{|l|}{ Excesso de peso } \\
\hline Sem excesso & 7.217 & $72,9(69,2-76,6)$ & 9.434 & $74,8(71,6-77,9)$ & 16.651 & $73,9(71,5-76,4)$ \\
\hline Com excesso & 2.507 & $27,1(23,4-30,8)$ & 3.012 & $25,2(22,1-28,4)$ & 5.519 & $26,1(23,6-28,5)$ \\
\hline
\end{tabular}

HBG: hemoglobina glicada; HDLc: colesterol associado à lipoproteína de alta densidade; LDLc: colesterol associado à lipoproteína de baixa densidade. 
Tabela 4

Odds ratio (OR) e intervalos de $95 \%$ de confiança (IC95\%) de morbidades em adolescentes brasileiros, segundo sexo e consumo de alimentos fora de casa. Estudo de Riscos Cardiovasculares em Adolescentes (ERICA), Brasil, 2013-2014.

\begin{tabular}{lcccccc}
\hline Morbidades & \multicolumn{2}{c}{ Modelo * $^{*}$} & \multicolumn{2}{c}{ Modelo 2 ** } & \multicolumn{2}{c}{ Modelo *** } \\
& OR & IC95\% & OR & IC95\% & OR & IC95\% \\
\hline Sexo masculino & & & & & & \\
Hipertensão arterial & 0,83 & $0,62-1,10$ & 0,82 & $0,61-1,10$ & 0,79 & $0,61-1,02$ \\
Hipercolesterolemia & 1,12 & $0,92-1,36$ & 1,06 & $0,88-1,27$ & 1,12 & $0,87-1,44$ \\
HDLc não desejável & 0,93 & $0,76-1,15$ & 0,95 & $0,77-1,18$ & 0,95 & $0,77-1,16$ \\
LDLc elevado & 1,02 & $0,79-1,33$ & 1,04 & $0,80-1,36$ & 0,97 & $0,74-1,27$ \\
Hipertrigliceridemia & 1,06 & $0,76-1,49$ & 1,03 & $0,74-1,44$ & 1,08 & $0,76-1,53$ \\
Hiperinsulinemia & 0,70 & $0,49-0,98$ & 0,70 & $0,50-0,98$ & 0,65 & $0,46-0,92$ \\
Hiperglicemia & 0,47 & $0,31-0,71$ & 0,48 & $0,32-0,73$ & 0,46 & $0,30-0,71$ \\
HBG elevada & 1,12 & $0,89-1,40$ & 1,17 & $0,94-1,46$ & 1,15 & $0,92-1,43$ \\
Excesso de peso & 1,11 & $0,89-1,39$ & 1,05 & $0,85-1,30$ & 1,04 & $0,84-1,30$ \\
Sexo feminino & & & & & & \\
Hipertensão arterial & 0,70 & $0,56-0,89$ & 0,72 & $0,57-0,92$ & 0,71 & $0,55-0,92$ \\
Hipercolesterolemia & 0,92 & $0,75-1,12$ & 0,88 & $0,72-1,08$ & 0,87 & $0,70-1,09$ \\
HDLc não desejável & 0,92 & $0,78-1,09$ & 0,95 & $0,82-1,11$ & 1,00 & $0,86-1,17$ \\
LDLc elevado & 1,01 & $0,86-1,19$ & 1,03 & $0,88-1,22$ & 1,05 & $0,88-1,25$ \\
Hipertrigliceridemia & 0,90 & $0,70-1,16$ & 0,89 & $0,70-1,13$ & 0,93 & $0,71-1,21$ \\
Hiperinsulinemia & 0,82 & $0,63-1,07$ & 0,85 & $0,67-1,09$ & 0,81 & $0,63-1,06$ \\
Hiperglicemia & 0,61 & $0,38-0,97$ & 0,64 & $0,41-1,02$ & 0,57 & $0,34-0,96$ \\
HBG elevada & 1,01 & $0,82-1,24$ & 1,04 & $0,84-1,28$ & 0,96 & $0,76-1,21$ \\
Excesso de peso & 0,92 & $0,72-1,19$ & 0,91 & $0,72-1,12$ & 0,95 & $0,75-1,20$ \\
\hline
\end{tabular}

HBG: hemoglobina glicada; HDLc: colesterol associado à lipoproteína de alta densidade; LDLc: colesterol associado à lipoproteína de baixa densidade.

* Modelo 1: idade;

** Modelo 2: Modelo 1 + rede de ensino da escola;

$* * *$ Modelo 3: Modelo $2+$ atividade física e horas de tela. 
Média de ingestão de energia, nutrientes e alimentos específicos e intervalos de 95\% de confiança (IC95\%), segundo consumo de alimentos fora de casa. Estudo de Riscos Cardiovasculares em Adolescentes (ERICA), Brasil, 2013-2014.

\begin{tabular}{|c|c|c|c|c|c|c|}
\hline \multirow[t]{3}{*}{ Variáveis de consumo } & \multicolumn{4}{|c|}{ Consumo de alimentos fora de casa } & \multicolumn{2}{|r|}{ Total } \\
\hline & \multicolumn{2}{|r|}{ Não } & \multicolumn{2}{|r|}{ Sim } & \multirow[b]{2}{*}{ Média } & \multirow[b]{2}{*}{$\%($ IC95\%) } \\
\hline & Média & $\%(I C 95 \%)$ & Média & $\%(I C 95 \%)$ & & \\
\hline Energia total (Kcal/dia) & $2.139,8$ & $2.085,4-2.194,2$ & $2.445,1$ & $2.350,0-2.540,2$ & $2.302,3$ & $2.235,5-2.369,1$ \\
\hline Açúcar de adição (\%VCT) & 13,5 & 0,0 & 14,7 & 0,0 & 14,2 & 0,0 \\
\hline Sódio (mg/1.000Kcal/dia) & $1.532,4$ & $1.508,8-1.556,0$ & $1.483,6$ & $1.466,3-1.500,9$ & $1.506,4$ & $1.071,1-1.118,4$ \\
\hline Potássio (mg/1.000Kcal/dia) & $1.102,8$ & $1.085,3-1.120,3$ & $1.087,7$ & $1.052,2-1.123,1$ & $1.094,7$ & $1.071,1-1.118,4$ \\
\hline Relação sódio/Potássio & 1,6 & 0,0 & 1,5 & 0,0 & 1,5 & 0,0 \\
\hline Fibras (g/dia) & 18,1 & 0,0 & 19,9 & 0,0 & 19,1 & 0,0 \\
\hline Frutas (g/dia) & 37,4 & 0,0 & 51,1 & 0,0 & 44,6 & 0,0 \\
\hline Verduras (g/dia) & 29,9 & 0,0 & 36,1 & 0,0 & 33,2 & 0,0 \\
\hline Arroz (g/dia) & 132,9 & 0,0 & 129,9 & 0,0 & 131,3 & 0,0 \\
\hline Feijão (g/dia) & 171,5 & 0,0 & 179,1 & 0,0 & 175,6 & 0,0 \\
\hline Sanduíches (g/dia) & 18,3 & 0,0 & 30,3 & 0,0 & 24,7 & 0,0 \\
\hline Bolos (g/dia) & 50,3 & 0,0 & 54,8 & 0,0 & 52,7 & 0,0 \\
\hline Sobremesas (g/dia) & 27,2 & 0,0 & 37,0 & 0,0 & 32,4 & 0,0 \\
\hline Chocolates (g/dia) & 60,1 & 0,0 & 73,1 & 0,0 & 67,0 & 0,0 \\
\hline Refrigerantes (mL/dia) & 216,1 & 0,0 & 261,9 & 0,0 & 240,5 & 0,0 \\
\hline
\end{tabular}

VCT: valor calórico total.

\section{Discussão}

Neste estudo de base escolar, realizado com 36.956 adolescentes brasileiros, encontrou-se uma relação inversa entre consumo de alimentos fora de casa e a ocorrência de hiperglicemia em ambos os sexos. No entanto, o consumo de alimentos fora de casa não mostrou associação com excesso de peso e outros biomarcadores para doenças crônicas (hemoglobina glicada, triglicerídeos, colesterol total, LDL-c e HDL-c).

Nossos achados estão de acordo com outros estudos transversais e longitudinais que avaliaram a relação entre consumo de alimentos fora de casa e excesso de peso e alterações em marcadores bioquímicos para doenças crônicas. Em 3.875 crianças chinesas de 7 a 17 anos, o consumo de alimentos fora de casa não mostrou associação com pressão arterial, hemoglobina glicada e proteína C-reativa 24 . Por outro lado, o consumo de alimentos preparados fora de casa por mais de duas vezes por semana por mulheres adultas australianas (26 a 36 anos) foi associado a maior nível de glicose e insulina séricos em comparação com as mulheres que consumiam somente uma vez por semana 7 . Entre adolescentes americanos, a maior frequência de aquisição de refeições em restaurantes do tipo fast food e com serviço de embalagem de comidas para levar ao domicílio (takeout) pela família está associada a maiores níveis de insulina e risco metabólico 6 .

A relação inversa da alimentos fora de casa com hiperglicemia chama atenção para os tipos de refeições que estão sendo consumidas dentro do domicílio. Entretanto, no presente estudo, foi visto um maior consumo de alimentos não saudáveis (sanduíches, sobremesas e refrigerantes), de calorias e de açúcar de adição nos adolescentes que consomem alimentos fora de casa. No geral, o consumo de açúcar de adição mostrou-se elevado tanto para aqueles adolescentes que consumiam alimentos fora de casa como naqueles que não consumiam, ultrapassando a recomendação de $10 \%$ do valor calórico total diário 25. No estudo de Azeredo et al. 26 foi encontrado um elevado consumo de doces, refrigerantes e biscoitos doces em adolescentes brasileiros, e menos de $30 \%$ dos avaliados consumiam vegetais. 
Também foi encontrado uma menor chance de ter hipertensão nos adolescentes do sexo feminino que comem fora de casa. Apesar disso, não encontramos diferenças no consumo de sódio entre adolescentes consumidores ou não consumidores de AFC. Além do consumo excessivo de sódio, o excesso de açúcar adicionado também está associado com o aumento de fatores adversos de saúde cardiovascular em crianças, especificamente níveis elevados de pressão diastólica e triglicérides 27.

Análises menos conservadoras, considerando os pontos de corte limítrofes para os marcadores bioquímicos (triglicerídeos $\geq 100 \mathrm{mg} / \mathrm{dL}$, colesterol total $\geq 150 \mathrm{mg} / \mathrm{dL}, \mathrm{LDL}-\mathrm{c} \geq 100 \mathrm{mg} / \mathrm{dL}$, insulina $\geq$ $15 \mathrm{mU} / \mathrm{L}$ ) não demonstraram diferenças nos resultados, com exceção da diferença na prevalência de hiperinsulinemia nos meninos, que foi maior naqueles que não se alimentavam fora de casa.

Nossos resultados devem ser vistos com cautela dada a natureza transversal do nosso estudo, contudo esses desfechos, na sua maioria, não eram conhecidos pelos estudantes. Outro ponto limitante é a classificação de indivíduos como consumidores de alimentos fora de casa, visto que se trata de uma amostra de adolescentes que ainda apresentam um consumo elevado de alimentos provenientes da escola. No entanto, a análise excluindo esse consumo indica não ser essa a explicação para os achados. Outra questão a ser considerada é a dificuldade em se estimar a ingestão de sódio via dieta, sendo o sódio urinário o padrão ouro para avaliação de sua ingestão dietética. No entanto, o método de múltiplos passos, como o usado para a coleta dos recordatórios no ERICA, fornece uma mensuração válida para a estimativa da ingestão de sódio em grupos populacionais 28.

Estudos que avaliem o consumo de alimentos fora de casa em adolescentes são limitados no Brasil. Nosso estudo destaca-se por usar uma amostra representativa de adolescentes das cinco regiões brasileiras, avaliando o hábito de consumir alimentos fora do lar e a mensuração dos biomarcadores para doenças crônicas não transmissíveis.

Os achados de maior prevalência de hipertensão e hiperglicemia em adolescentes que não consumiram alimentos fora de casa são de extrema importância para ampliar o olhar para o tipo de alimentação que vem sendo realizada por esse grupo populacional. Crianças e adolescentes que relataram consumir fast food apresentaram uma baixa qualidade nutricional no restante da dieta (sem incluir os alimentos do tipo fast food) ${ }^{29}$, além de apresentarem um padrão alimentar mais inadequado ${ }^{30}$. Se a alimentação do domicílio é adquirida em locais com alimentos não saudáveis, certamente influenciará o maior consumo de açúcares adicionados, sendo esse um fator que pode levar à obesidade 31,32.

No nosso estudo, nós observamos uma alta prevalência de excesso de peso nos adolescentes, independente do consumo de alimentos fora de casa. Cunha et al. 33 estudaram padrões alimentares dentro e fora de casa de adolescentes brasileiros e encontraram que a ingestão alimentar média em casa foi maior do que a ingestão de alimentos fora de casa, e três padrões alimentares semelhantes foram identificados tanto em casa como fora de casa: o "padrão tradicional”, o "padrão pão e manteiga" e o "padrão ocidental" 33. Corroborando com os nossos achados, apenas o "padrão ocidental” domiciliar foi positivamente associado aos escores-z do IMC $(b=0,0006 ; p<0,001) 33$, indicando que estratégias de estímulo a hábitos alimentares saudáveis entre os adolescentes devem abordar tanto escolhas alimentares caseiras como fora de casa 34 .

O tipo de escola foi incluído em nossa análise como um proxy do nível socioeconômico, partindo do pressuposto que alunos de escolas privadas apresentam maior nível socioeconômico. Algumas diferenças foram observadas entre os estudantes de escolas públicas e privadas. Adolescentes de escolas públicas que consumiram alimentos fora de casa apresentaram menor probabilidade de terem hiperinsulinemia e hiperglicemia entre os meninos e menor probabilidade de apresentar hipertensão arterial e hiperglicemia entre as meninas das escolas públicas.

Tendo em vista que parte do consumo de alimentos fora de casa é decorrente da alimentação escolar, foram avaliados a oferta e o consumo da alimentação oferecida na escola pelos adolescentes, e encontramos que o consumo de alimentação escolar é similar entre os que consomem e não consomem alimentos fora de casa. Por outro lado, a frequência desse consumo é três vezes maior nos adolescentes de escola pública do que nos adolescentes de escolas privadas (61,7\%; IC95\%: 58,8-64,7 vs. 21,4\%; IC95\%: 16,0-26,7), o que explica, em parte, nossos achados, uma vez que a alimentação escolar contribui de forma importante para alimentação dos estudantes de escolas públicas.

Sendo este trabalho de base escolar, enfatiza-se a importância da alimentação na escola, já que se sabe que um fornecimento de alimentos saudáveis, como frutas e legumes, na refeição ofertada pela escola, pode propiciar um melhor consumo desse grupo alimentar 35 . Em concordância com 
essa afirmação, análises estratificadas pelo tipo de escola mostraram que a ingestão média calórica e o maior consumo de frutas e feijão foram observados somente entre os adolescentes de escolas públicas, sugerindo a influência da alimentação da escola na dieta desses indivíduos. Por outro lado, é importante destacar o maior consumo de açúcar de adição, sanduíches, sobremesas e refrigerantes entre esses adolescentes, embora a maioria dos itens avaliados tenham apresentado maior média de consumo entre adolescentes de escolas privadas, com exceção dos refrigerantes, que não apresentaram diferença, e de arroz e feijão, que tiverem maior consumo entre adolescentes de escolas públicas.

No estudo de Alves et al. 36, que avaliaram os padrões alimentares de adolescentes participantes do ERICA, foi encontrado que os estudantes de escolas privadas apresentaram maior adesão ao padrão alimentar não saudável e menor adesão ao padrão tradicional 36.

O consumo de calorias, açúcar de adição, sanduíches, sobremesas e refrigerantes foi maior nos adolescentes que consumiam alimentos fora de casa, mas também foi mais frequente o consumo da alimentação escolar. Assim, o papel protetor da alimentação fora de casa em indicadores bioquímicos nos adolescentes pode ser em função de um maior consumo da alimentação escolar. A educação alimentar para adolescentes brasileiros deve enfatizar o estímulo ao consumo da alimentação ofertada pela escola.

\section{Colaboradores}

S. R. Morais, I. N. Bezerra e C. M. A. C. Vergara contribuíram na concepção, delineamento e condução do estudo, redação e revisão do artigo. A. M. Souza e R. Sichieri contribuíram na concepção e delineamento do estudo e revisão crítica do conteúdo.

\section{Informações adicionais}

ORCID: Suelyne Rodrigues de Morais (0000-00019827-8219); Ilana Nogueira Bezerra (0000-00022072-0123); Amanda de Moura Souza (0000-00028962-798X); Clarice Maria Araújo Chagas Vergara (0000-0003-1709-9951); Rosely Sichieri (00000001-5286-5354).

\section{Agradecimentos}

Agradecemos aos profissionais e pesquisadores, às escolas e aos alunos participantes da pesquisa que permitiram a realização deste estudo. À Coordenação de Aperfeiçoamento de Pessoal de Nível Superior pela concessão de bolsa de mestrado.

\section{Referências}

1. Donin AS, Nightingale CM, Owen CG, Rudnicka AR, Cook DG, Whincup PH. Takeaway meal consumption and risk markers for coronary heart disease, type 2 diabetes and obesity in children aged 9-10 years: a cross-sectional study. Arch Dis Child 2018; 103:431-6.

2. Mancino L, Todd JE, Guthrie J, Lin BH. Food away from home and childhood obesity. Curr Obes Rep 2014; 3:459-69.

3. Bezerra IN, Curioni C, Sichieri R. Association between eating out of home and body weight. Nutr Rev 2012; 70:65-79.

4. Pereira MA, Kartashov AI, Ebbeling CB, Van Horn L, Slattery ML, Jacobs Jr. DR, et al. Fastfood habits, weight gain, and insulin resistance (the CARDIA study): 15-year prospective analysis. Lancet 2005; 365:36-42.

5. Duffey KJ, Gordon-Larsen P, Steffen LM, Jacobs Jr. DR, Popkin BM. Regular consumption from fast food establishments relative to other restaurants is differentially associated with metabolic outcomes in young adults. J Nutr 2009; 139:2113-8. 
6. Fulkerson JA, Farbakhsh K, Lytle L, Hearst MO, Dengel DR, Pasch KE, et al. Away-fromhome family dinner sources and associations with weight status, body composition, and related biomarkers of chronic disease among adolescents and their parents. J Am Diet Assoc 2011; 111:1892-7.

7. Smith KJ, Blizzard L, McNaughton SA, Gall SL, Dwyer T, Venn AJ. Takeaway food consumption and cardio-metabolic risk factors in young adults. Eur J Clin Nutr 2012; 66:577-84.

8. Burke SJ, McCarthy SN, O’Neill JL, Hannon EM, Kiely M, Flynn A, et al. An examination of the influence of eating location on the diets of Irish children. Public Health Nutr 2007; 10:599-607.

9. Bezerra IN, Souza ADM, Pereira RA, Sichieri R. Consumo de alimentos fora do domicílio no Brasil. Rev Saúde Pública 2013; 47:200s-11s.

10. Lewis MN, Shatat IF, Phillips SM. Screening for hypertension in children and adolescents: methodology and current practice recommendations. Front Pediatr 2017; 5:51.

11. Kumar S, Kelly AS. Review of childhood obesity: from epidemiology, etiology, and comorbidities to clinical assessment and treatment. Mayo Clin Proc 2017; 92:251-65.

12. Bloch KV, Cardoso MA, Sichieri R. Study of Cardiovascular Risk Factors in Adolescents (ERICA): results and potentiality. Rev Saúde Pública 2016; 50 Suppl 1:2s.

13. Silva TLN, Klein CH, Souza AM, Barufaldi LA, Abreu GDA, Kuschnir MCC, et al. Response rate in the Study of Cardiovascular Risks in Adolescents - ERICA. Rev Saúde Pública 2016; 50 Suppl 1:3s.

14. Vasconcellos MTL, Silva PLN, Szklo M, Kuschnir MCC, Klein CH, Abreu GD, et al. Sampling design for the Study of Cardiovascular Risks in Adolescents (ERICA). Cad Saúde Pública 2015; 31:921-30.

15. Bloch KV, Szklo M, Kuschnir MC, Abreu GA, Barufaldi LA, Klein CH, et al. The Study of Cardiovascular Risk in Adolescents - ERICA: rationale, design and sample characteristics of a national survey examining cardiovascular risk factor profile in Brazilian adolescentes. BMC Public Health 2015; 15:94.

16. Onis MD, Onyango AW, Borghi E, Siyam A, Nishida C, Siekmann J. Development of a WHO growth reference for school-aged children and adolescents. Bull World Health Organ 2007; 85:660-7.

17. Xavier HT, Izar MC, Faria Neto JR, Assad $\mathrm{MH}$, Rocha VZ, Sposito AC, et al. V Diretriz Brasileira de Dislipidemias e Prevenção da Aterosclerose. Arq Bras Cardiol 2013; 101(4 Suppl 1):1-20.

18. American Diabetes Association. Classification and diagnosis of diabetes: standards of medical care in diabetes-2018. Diabetes Care 2018; 41 Suppl 1:S13-27.
19. Giuliano IDCB, Caramelli B, Pellanda LC, Duncan BB, Mattos S, Fonseca FAH. I Diretriz de Prevenção da Aterosclerose na Infância e na Adolescência. Arq Bras Cardiol 2005; 85 Suppl 6:3-36.

20. Barufaldi LA, Abreu GDA, Veiga GVD, Sichieri R, Kuschnir MCC, Cunha D, et al. Programa para registro de recordatório alimentar de 24 horas: aplicação no Estudo de Riscos Cardiovasculares em Adolescentes. Rev Bras Epidemiol 2016; 19:464-68.

21. Moshfegh AJ, Rhodes DG, Baer DJ, Murayi T, Clemens JC, Rumpler WV, et al. The US Department of Agriculture Automated MultiplePass Method reduces bias in the collection of energy intakes. Am J Clin Nutr 2008; 88:32432.

22. Hallal PC, Knuth AG, Cruz DKA, Mendes MI, Malta DC. Prática de atividade física em adolescentes brasileiros. Ciênc Saúde Colet 2010; 15:3035-42.

23. Departamento de Atenção Básica, Secretaria de Atenção à Saúde, Ministério da Saúde. Guia alimentar para a população brasileira. 2a $\mathrm{Ed}$. Brasília: Ministério da Saúde; 2014.

24. Dong F, Howard AG, Herring AH, Thompson AL, Adair LS, Popkin BM, et al. Longitudinal associations of away-from-home eating, snacking, screen time, and physical activity behaviors with cardiometabolic risk factors among Chinese children and their parents. Am J Clin Nutr 2017; 106:168-78.

25. World Health Organization. Guideline: sugars intake for adults and children. Geneva: World Health Organization; 2015.

26. Azeredo CM, Rezend LFM, Canella DS, Claro RM, Castro IRR, Carmo Luiz O, et al. Dietary intake of Brazilian adolescents. Public Health Nutr 2015; 18:1215-24.

27. Kell KP, Cardel MI, Bohan Brown MM, Fernández JR. Added sugars in the diet are positively associated with diastolic blood pressure and triglycerides in children. Am J Clin Nutr 2014; 100:46-52.

28. Rhodes DG, Murayi T, Clemens JC, Baer DJ, Sebastian RS, Moshfegh AJ. The USDA Automated Multiple-Pass Method accurately assesses population sodium intakes. Am J Clin Nutr 2013; 97:958-64.

29. Poti JM, Duffey KJ, Popkin BM. The association of fast food consumption with poor dietary outcomes and obesity among children: is it the fast food or the remainder of the diet? Am J Clin Nutr 2014; 99:162-71.

30. Maia EG, Silva LESD, Santos MAS, Barufaldi LA, Silva SUD, Claro RM. Dietary patterns, sociodemographic and behavioral characteristics among Brazilian adolescents. Rev Bras Epidemiol 2018; 21:e180009. 
31. Gustafson A, Jilcott PS, McDonald J, Ford H, Connelly P, Gillespie R, et al. Direct effects of the home, school, and consumer food environments on the association between food purchasing patterns and dietary intake among rural adolescents in Kentucky and North Carolina, 2017. Int J Environ Res Public Health 2017; 14:E1255.

32. Grimes CA, Riddell LJ, Campbell KJ, Nowson CA. Dietary salt intake, sugar-sweetened beverage consumption, and obesity risk. Pediatrics 2013; 131:14-21.

33. Cunha DB, Bezerra IN, Pereira RA, Sichieri R. At-home and away-from-home dietary patterns and BMI z-scores in Brazilian adolescents. Appetite 2018; 120:374-80.
34. Claro RM, Baraldi LG, Martins APG, Bandoni DH, Levy RB. Trends in spending on eating away from home in Brazil, 2002-2003 to 2008-2009. Cad Saúde Pública 2014; 30:141826.

35. Miller N, Reicks M, Redden JP, Mann T, Mykerezi E, Vickers, Z. Increasing portion sizes of fruits and vegetables in an elementary school lunch program can increase fruit and vegetable consumption. Appetite 2015; 91:426-30

36. Alves MA, Souza AM, Barufaldi LA, Tavares BM, Bloch KV, Vasconcelos FAG. Padrões alimentares de adolescentes brasileiros por regiões geográficas: análise do Estudo de Riscos Cardiovasculares em Adolescentes (ERICA). Cad Saúde Pública 2019; 35:e0153818. 


\section{Abstract}

The study aimed to assess the relationship between food consumption away from home and alterations in biomarkers for chronic noncommunicable diseases in Brazilian adolescents. This cross-sectional study used data from the Study of Cardiovascular Risk Factors in Adolescents (ERICA), conducted in 36,956 adolescents in 2013/2014. The relationship between food consumption away from home and each target outcome (hypertriglyceridemia, hypercholesterolemia, hyperglycemia, high glycated hemoglobin, and hyperinsulinemia) was tested with logistic regression models adjusted for age, school system (public versus private), physical activity, and screen time. Data on food consumption were obtained with a 24-hour diet recall (24HR), analyzing consumption of energy, added sugar, sodium, potassium, fiber, fruits, vegetables, rice, beans, sandwiches, cakes, dessert, chocolates, and sodas. The results showed that 53.2\% of adolescents consumed foods away from home. Eating away from home showed an inverse relationship with hyperinsulinemia $(O R=0.65$; 95\%CI: 0.46-0.92) and hyperglycemia $(O R=0.46$; 95\%CI: 0.30-0.71) in boys and hypertension (OR $=0.71 ; 95 \%$ CI: 0.55-0.92) and hyperglycemia $(O R$ $=0.57 ; 95 \%$ CI: 0.34-0.96) in girls. However, the consumption of calories, added sugar, sandwiches, desserts, and sodas was higher in adolescents that consumed foods away from home. The protective role of eating away from home, as measured by biochemical indicators in adolescents, may be a function of higher consumption of school meals, which was more frequent among adolescents that consumed food away from home, thus highlighting the importance of encouraging consumption of school meals.

Feeding Behavior; Life Style; Hypertension; Diabetes Mellitus; Noncommunicable Diseases

\section{Resumen}

El objetivo de la investigación fue evaluar la relación entre el consumo de alimentos fuera de casa $y$ las alteraciones en biomarcadores de enfermedades crónicas en adolescentes brasileños. Se trata de un estudio transversal, en el que se utilizaron los datos del Estudio de Riesgos Cardiovasculares en Adolescentes (ERICA), realizado con 36.956 adolescentes, en 2013/2014. La relación entre consumir alimentos fuera de casa y cada resultado de interés (hipertrigliceridemia, hipercolesterolemia, hiperglucemia, elevada hemoglobina glicada e hiperinsulinemia) se probó mediante modelos de regresión logística ajustados por edad, red de enseñanza de la escuela, actividad física y tiempo de uso de pantallas. Se obtuvieron datos del consumo alimentario mediante la aplicación del recordatorio referente a las $24 \mathrm{~h}$ anteriores $(R 24 \mathrm{~h})$, analizando el consumo de energía, azúcar añadido, sodio, potasio, fibras, frutas, verduras, arroz, frijoles, sándwiches, bizcochos, postres, chocolates y refrescos. Se encontró que un 53,2\% de los adolescentes consumían alimentos fuera de casa. La alimentación fuera de casa presentó una relación inversa con la hiperinsulinemia $(O R=0,65$; IC95\%: 0,46-0,92) e hiperglicemia $(O R=0,46$; IC95\%: 0,30-0,71) entre chicos, e hipertensión (OR $=0,71$; IC95\%: 0,55-0,92) e hiperglicemia $(\mathrm{OR}=$ 0,57; IC95\%: 0,34-0,96) entre chicas. Sin embargo, el consumo de calorías, azúcar añadido, sándwiches, postres y refrescos fue mayor en los adolescentes que consumían alimentos fuera de casa. El papel protector de la alimentación fuera de casa en indicadores bioquímicos en los adolescentes puede ser en función de un mayor consumo de merienda escolar, más frecuente entre los adolescentes que consumian alimentos fuera de casa, destacando la importancia del estímulo al consumo de la merienda escolar.

Conducta Alimentaria; Estilo de Vida;

Hipertensión; Diabetes Mellitus; Enfermedades

No Transmisibles
Recebido em 13/Nov/2019

Versão final reapresentada em 19/Mai/2020

Aprovado em 15/Jun/2020 